\title{
PACEMAKER AND DEFIBRILLATOR TEACHING SYSTEM WITH CARELINK AND HOMEMONITORING REMOTE PATIENT MONITORING
}

\author{
Haber T, Holzer F, Ismer B \\ Peter Osypka Institute for Pacing and Ablation at \\ Offenburg University of Applied Sciences, Germany \\ poi@hs-offenburg.de
}

\begin{abstract}
Special implant connection module was developed to combine full features of two commercial heart rhythm simulators, ARSI-4 and Intersim II, into a master-slave teaching system. Seven workstations were equipped with the Carelink and Homemonitoring remote patient monitoring systems. This combination enables invitro training of physicians, nurses and students in pacemaker and defibrillator measurements during implantation and individual programming in the follow-up. Thus, extended sets of arrhythmias and electrode problems can be used to simulate problems and their solutions in a wide range of the clinical routine.
\end{abstract}

Keywords: heart rhythm simulator, remote patient monitoring, teaching system,

\section{Introduction}

Single, dual and three chamber pacing or defibrillator therapy is established in patients suffering from bradycardia, tachycardia and cardiac desynchronization. In all of them, individualization of sensing, pacing and hemodynamic implant parameters is mandatory. It has to be performed postoperatively and during follow-up.
Depending on the individual situation, lots of parameters have to be considered in order to achieve best clinical outcome. This requires special experience and expertise of both, the attending physicians and the supporting implant companies employees. Their in-vitro training in pacemaker measuring and programming can be completed using heart rhythm simulators.

\begin{abstract}
Methods
Master-slave teaching system was established supplying seven stations. Each of them was equipped with wireless local area network computer providing accounts of the Homemonitoring (Biotronik; Berlin, Germany) /1, 2/ and Carelink (Medtronic, Minneapolis, USA) /3/ remote patient monitoring systems. To enable in-vitro training, several implants with end of life status were used after connecting external batteries and completing the power on reset procedure (Fig. 2). Special implant connection module was developed to provide the defibrillators and pacemakers with right atrial, right and left ventricular intracardial electrograms simulated by either the ARSI-4 or the Intersim II /4, 5/ heart rhythm simulator (Fig. 1).
\end{abstract}

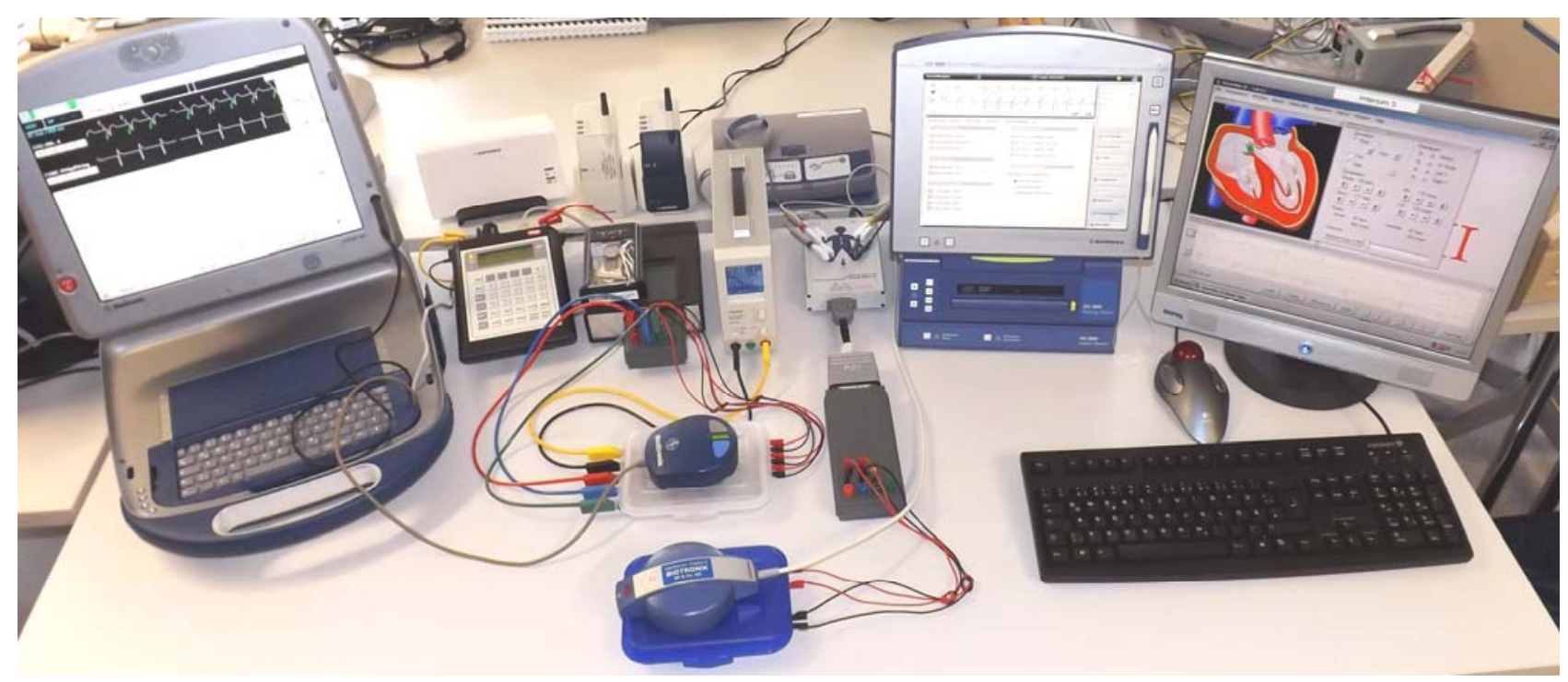

Figure 1: Developing special implant connection modules, commercial ARSI-4 and Intersim II heart rhythm simulators were combined in to a pacemaker and defibrillator teaching system and equipped with Carelink (Medtronic, Minneapolis, USA) and Homemonitoring (Biotronik, Berlin, Germany) remote patient monitoring. 


\section{Results}

The combination of ARSI-4 and Intersim II heart rhythm simulators in a single teaching system allows quick access to several heart rhythms with an increased quality of electrode electrograms. Thus, in-vitro training of physicians, nurses and students in pacemaker and defibrillator measurement and programming can be offered with extended sets of artificial arrhythmias as well as teaching of critical situations during pacemaker implantation and follow-up.

As an advantage of the combination of the two simulators with workstations, all results of the Internet based remote patient monitoring systems Carelink and Homemonitoring can be checked online, immediately. Additional screenshot software can be utilized to document the pacemaker and defibrillator behaviour as well as malfunctions of the implants due to inadequate electrode signals or inadequate programming. Using the features of the Windows graphical interface, capture software can be used to produce screenshots and videos for PowerPoint presentations.

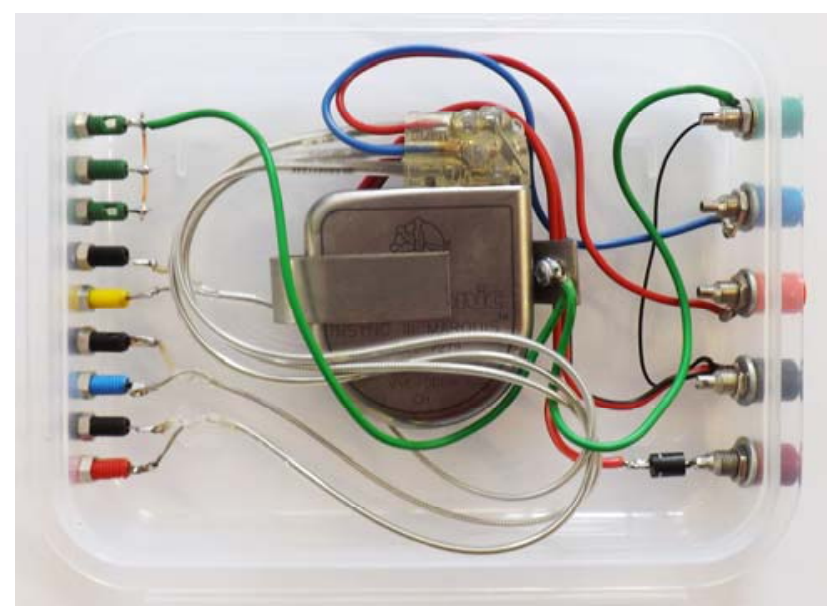

Figure 2: Example of full functional Medtronic Insync III Marquis implantable biventricular defibrillator system prepared to be supplied with external power assembled into an isolating box. After completing the power on reset procedure it can be used for training of physicians, nurses and students. As special advantage, this implant can also be used for teaching all features of the actual Carelink remote patient monitoring.

\section{Discussion}

Combining two commercial heart rhythm simulators, ARSI-4 and Intersim II, in-vitro training of physicians, nurses and students in pacemaker and defibrillator measurements during implantation and individual programming during follow-up can be offered with an extended set of artificial arrhythmias and electrode problems such as

- $\quad$ several types and rates of ventricular tachycardias and ventricular fibrillation

- several types and rates of atrial fibrillation

- several types and rates of atrial flutter
- AV nodal and AV reentrant tachycardias with different rates and conditions of termination

- several problems of the right atrial as well as the right and left ventricular electrodes with extended properties of artifacts, noise, alternating current hum and isolation defects

Furthermore, using two different simulators, more implant features can be studied within one session. As a special advantage, all results of the Internet based remote patient monitoring can be checked immediately. Screenshot and video capture software can be utilized to document the pacemaker and defibrillator behaviour, malfunctions and episodes and to produce videos for PowerPoint presentations.

\section{Conclusions}

Developing implant connection module for two commercial heart rhythm simulators and equipping the required workstations with Carelink and Homemonitoring accounts, in-vitro training in the field of antibradycardic, antitachycardic and cardiac resynchronization therapy can be performed by simulating problems and their solutions in a wide range of the clinical routine.

\section{Bibliography}

[1] Theuns DAMJ, Res JCJ. Jordaens LJ (2003) Home monitoring in ICD therapy: future perspective. Europace 5: 139-142, doi:10.1053/eupc.2002.0302

[2] Perings SM, Bläse I, Marx R, Plehn G, Müller C, Trappe HJ, Perings C (2008) Register zur verzahnten Nachsorge von ICD-Patienten durch Home Monitoring: Erfahrungen mit den ersten Patienten im 1-Jahres-Follow-up. Austrian Journal of Cardiology 15 (1-2), 17-22

[3] Brachmann J, Böhm M, Rybak K, Klein G, Butter C, Klemm H, Schomburg R, Siebermair J, Israel C, Sinha AM, Drexler H; OptiLink HF Study Executive Board and Investigators (2011) Fluid status monitoring with a wireless network to reduce cardiovascular-related hospitalizations and mortality in heart failure: rationale and design of the OptiLink HF Study (Optimization of Heart Failure Management using OptiVol Fluid Status Monitoring and CareLink). Eur J Heart Fail. 13 (7): 796-804. doi: 0.1093/eurjhf/hfr045. Epub 2011 May 8

[4] Description of the interactive Intersim II heart rhythm simulator. http://www.lang-ib.de

[5] Ismer B (2012) Zum Nutzen elektronischer Herzrhythmussimulatoren für die Herzschrittmacher- und Defibrillatortherapie. Beiträge aus Forschung und Technik: 29-31, Hochschule Offenburg 